\title{
The circumstellar dust shell of the post-AGB star HD $161796^{\star}$
}

\author{
S. N. Hoogzaad ${ }^{1}$, F. J. Molster ${ }^{2}$, C. Dominik ${ }^{1}$, L. B. F. M. Waters ${ }^{1,3}$, M. J. Barlow ${ }^{4}$, and A. de Koter ${ }^{1}$ \\ 1 Astronomical Institute "Anton Pannekoek", University of Amsterdam, Kruislaan 403, 1098 SJ Amsterdam, \\ The Netherlands \\ 2 ESTEC/ESA, RSSD-ST, Keplerlaan 1, 2201 AZ Noordwijk, The Netherlands \\ 3 Instituut voor Sterrenkunde, K.U. Leuven, Celestijnenlaan 200B, 3001 Heverlee, Belgium \\ 4 Department of Physics and Astronomy, University College London, Gower Street, London WC1E6BT, UK
}

Received 13 December 2001 / Accepted 14 March 2002

\begin{abstract}
We have modeled the complete optical to millimeter spectrum of the Post-Asymptotic Giant Branch (Post-AGB) star HD 161796 and its circumstellar dust shell. A full 2-200 $\mu \mathrm{m}$ spectrum taken with the Infrared Space Observatory was used to constrain the dust properties. A good fit is achieved using only 4 dust components: amorphous silicates, the crystalline silicates forsterite and enstatite, and crystalline water ice, contributing respectively about $63,4,6$ and $27 \%$ to the total dust mass. The different dust species were assumed to be co-spatial but distinct, resulting in different temperatures for the different grain populations. We find a temperature for the crystalline $\mathrm{H}_{2} \mathrm{O}$ ice of $70 \mathrm{~K}$, which is higher than thermal equilibrium calculations of pure $\mathrm{H}_{2} \mathrm{O}$ ice would give. This implies that the ice must be formed as a mantle on top of an (amorphous) silicate core. In order to form $\mathrm{H}_{2} \mathrm{O}$ ice mantles the mass loss rate must exceed some $5 \times 10^{-5} M_{\odot} \mathrm{yr}^{-1}$. With a water-ice fraction of $27 \%$ a lower limit for the gas to dust mass ratio of 270 is found. At a distance of $1.2 \mathrm{kpc}$ (Skinner et al. 1994) and adopting an outflow velocity of $15 \mathrm{~km} \mathrm{~s}^{-1}$ (Likkel et al. 1991) an AGB mass loss rate of $\left(5.1 \times 10^{-4} M_{\odot} \mathrm{yr}^{-1}\right)$ is found, which lasted 900 years and ended 430 years ago. During this phase a total of $0.46 M_{\odot}$ was expelled. The mass loss rate was high enough to account for the presence of the $\mathrm{H}_{2} \mathrm{O}$ ice.
\end{abstract}

Key words. stars: circumstellar matter, AGB and post-AGB - stars: individual: HD 161796 - infrared: ISM ISM: lines and bands

\section{Introduction}

Asymptotic Giant Branch (AGB) stars are stars with a Main Sequence (MS) mass between $0.8-8 M_{\odot}$ at the end of their life. These stars are characterised by high mass loss rates $\left(10^{-8}-10^{-3} M_{\odot} \mathrm{yr}^{-1}\right)$ through a slowly expanding $\left(v_{\exp }=10-20 \mathrm{~km} \mathrm{~s}^{-1}\right)$ dusty outflow. At the end of the AGB, such stars have lost $40-90 \%$ of their MS mass. Because mass loss dominates stellar evolution timescales on the AGB, it is necessary to understand the mass loss process, if we want to understand their evolution. The remnants of this huge mass loss episode, the circumstellar gas and dust shells around post-AGB stars, give us valuable insight into the physical processes that took place at the end of the AGB. Furthermore, AGB stars provide about $50 \%$ of the mass returned into the interstellar medium (ISM), so also for a better understanding of the

\footnotetext{
Send offprint requests to: L. B. F. M. Waters, e-mail: rensw@astro.uva.nl

* Based on observations with ISO, an ESA project with instruments funded by ESA Member States (especially the PI countries: France, Germany, The Netherlands and the UK) and with the participation of ISAS and NASA.
}

dust-cycle in galaxies it is important to investigate the formation and properties of dust in AGB stars.

In this paper we study HD 161796, a high latitude F3Ib (Fernie \& Garrison 1984) post-AGB star with a detached circumstellar dust envelope (Parthasarathy \& Pottasch 1986; Skinner et al. 1994). Its distance is estimated to be $1.2 \mathrm{kpc}$ (Skinner et al. 1994) The central star has a temperature of $6750 \mathrm{~K}$ and the extinction to the star is small $(E(B-V)=0.19$; Van Winckel, priv. comm.). The detection of an OH-maser (Likkel 1989) shows that the circumstellar environment is oxygen-rich. This was confirmed by Justtanont et al. (1992) with the detection of the characteristic 10 and 20 micron amorphous silicate dust features. The expansion velocity, based on the COline emission, is $15 \mathrm{~km} \mathrm{~s}^{-1}$ with wings up to $40 \mathrm{~km} \mathrm{~s}^{-1}$ (Likkel et al. 1991).

In 1996 and 1997 the Infrared Space Observatory (ISO; Kessler et al. 1996) observed HD 161796 in the wavelength range from 2.4 to $197 \mu \mathrm{m}$. These spectra were already discussed qualitatively by Barlow (1998) and Molster (2000), and are characterized by the presence of a large number of broad and narrow emission bands due to amorphous 
and crystalline silicates and crystalline $\mathrm{H}_{2} \mathrm{O}$ ice. In this paper we quantitatively model the complete spectrum of the star to determine the properties of the circumstellar dust envelope. We will also derive estimates for the conditions under which the large amount of (crystalline) water ice, which we will show in this paper is present around HD 161796, was formed.

This paper is organized as follows: in Sect. 2 we describe the ISO-observations and shortly discuss the spectrum. In Sect. 3 we use a full radiative transfer model (MODUST) to determine the properties of the dust shell. Section 4 describes the results of our modeling and the constraints we can put on various model parameters. We discuss the formation of water-ice mantles onto (amorphous) silicate cores in Sect. 5. The main results of the paper are summarized in the final section.

\section{The spectrum}

The ISO spectrum of HD 161796 has been extensively discussed in Molster (2000) and Molster et al. (2002b); we will therefore only briefly discuss the reduction of and the features present in the ISO-spectrum. In order to model the complete spectrum of HD 161796 we extend the wavelength range with International Ultraviolet Explorer (IUE) data and optical data from Fernie (1983). We also included the continuum measurement at 1.3 millimeter (Walmsley et al. 1991).

\subsection{Observations}

In total four spectra of HD 161796 were obtained with ISO: two Short Wavelength Spectrometer (SWS; de Graauw et al. 1996) spectra covering the full SWS wavelength range $(2.4-45 \mu \mathrm{m})$ in revolution 71 and 342 , one high spectral resolution SWS spectrum from 29.0 to $45.2 \mu \mathrm{m}$ during revolution 521, and one Long Wavelength Spectrometer (LWS; Clegg et al. 1996) spectrum covering its full spectral range $(43-197 \mu \mathrm{m})$ in revolution 80 . In total we have a continuous spectrum from 2.4 up to $197 \mu \mathrm{m}$. In the final spectrum the SWS data from revolution 71 was not used, although it was used to check for artifacts in the spectrum.

The SWS spectra were reduced using the SWS offline processing software, version 7.0. Each detector was checked for irregularities and if necessary bad sections of the spectrum of individual detectors were removed from the data. The main fringes were removed in the band 3 datasets. Finally, the 12 detectors of each sub-band were flat fielded and a sigma clipping procedure was used to remove deviating points; finally, the results were rebinned. Hereafter the different sub-bands were shifted or multiplied to match each other, when respectively the dark current or the flux calibration was expected to be the main source of error.

The LWS spectrum was reduced by R. Sylvester using the LWS off-line processing software (version 7.0). The

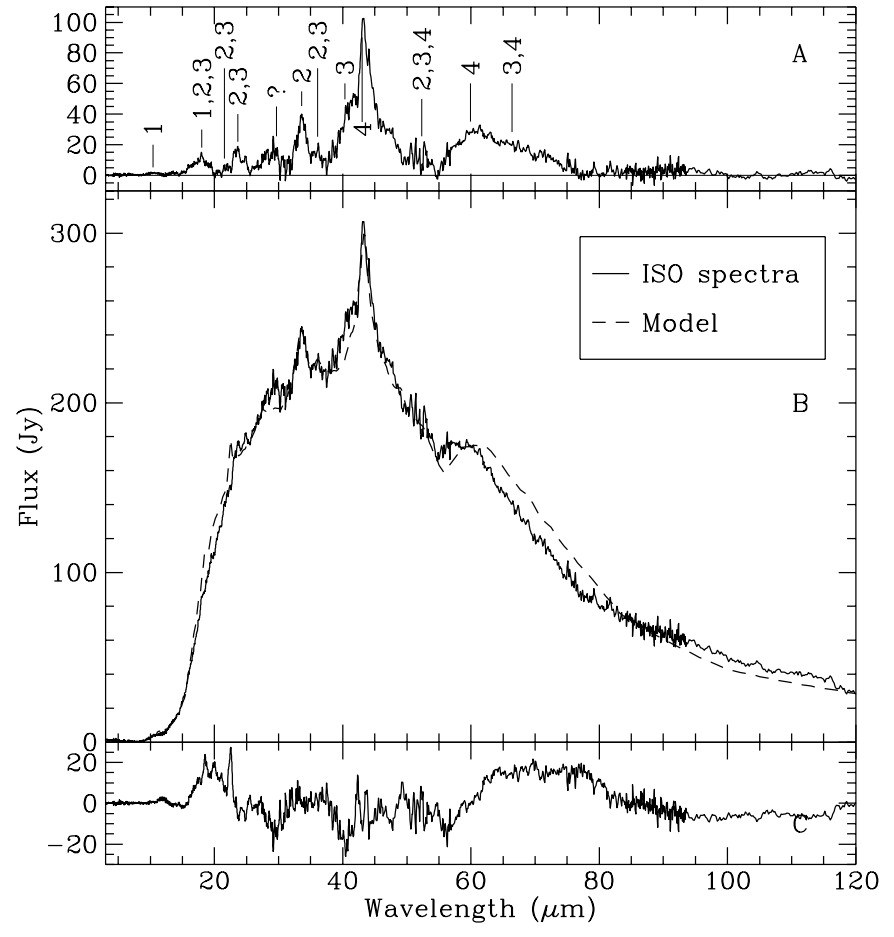

Fig. 1. Panel a) A pseudo-continuum has been subtracted from the spectrum (shown in panel B) to enhance the features. $1=$ amorphous silicate, $2=$ forsterite, $3=$ enstatite, $4=$ crystalline water ice (see Molster et al. 2002a for a more detailed description of the identifications). Panel b) A fit of the full radiative transfer model (dashed line) to the measured spectrum of HD 161796 (solid line). Panel c) The residual between the observed spectrum and the model is shown.

data from the different scans were averaged after sigmaclipping to remove deviating data points caused by cosmicray hits. The ten sub-spectra from the ten detectors were rescaled by small factors to give consistent fluxes in regions of overlap and merged for the final spectrum. The high galactic latitude of HD 161796 implies that contamination by cold interstellar dust in the LWS beam is negligible.

The reduced LWS and SWS spectra matched very well in their overlapping region around $45 \mu \mathrm{m}$, which is an indication for the quality of the data reduction process. The final spectrum can be found in Figs. 1 and 2; in Fig. 2 we also included the UV, optical and radio continuum measurements of the star.

\subsection{A global description of the infrared spectrum}

The ISO spectrum shows a broad continuum on top of which are two strong features at 43 and $60 \mu \mathrm{m}$. This points to the presence of a large amount of crystalline water ice. The narrow features in the 23, 28, 33 and 40 micron complexes, indicate the presence of the crystalline silicates (Molster et al. 2002b). The wavelength position of these features is in agreement with very Fe-poor silicates, i.e. enstatite and forsterite (see e.g. Jäger et al. 1998). Broad amorphous silicate features around $10 \mu \mathrm{m}$ and $18 \mu \mathrm{m}$ are also present. 


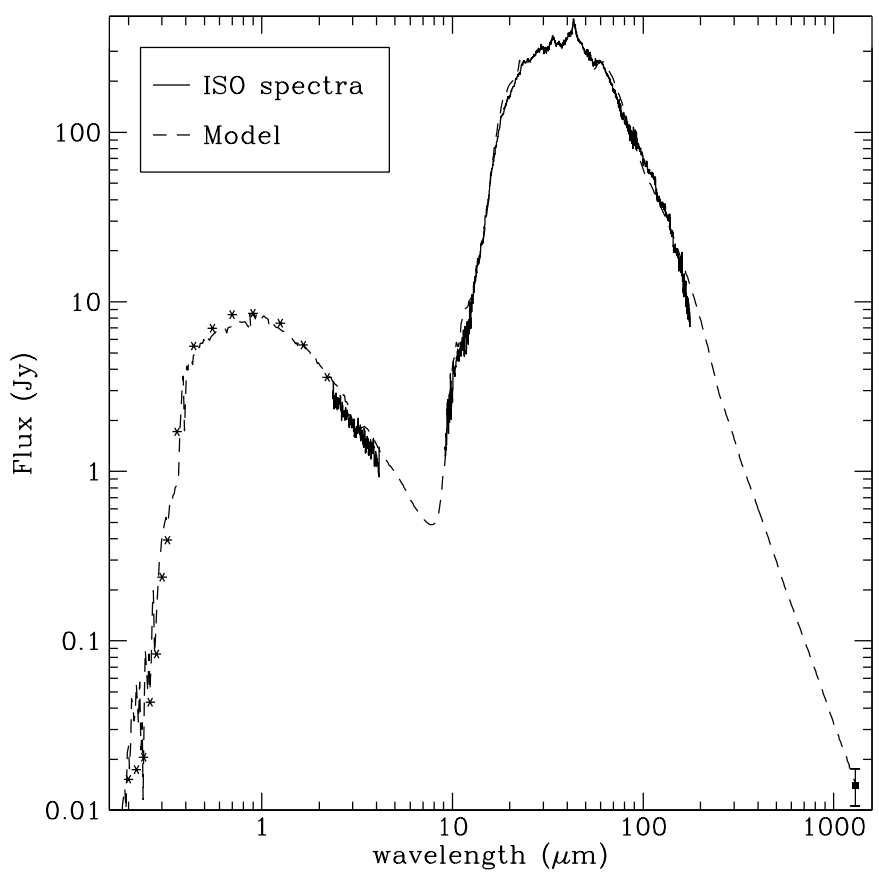

Fig. 2. A fit of the full radiative transfer model (dashed line) to the measured spectrum of HD 161796 (solid line) on log scales. The asterisks are the UV and optical data, and the observation at $1.3 \mathrm{~mm}$ is taken from Walmsley et al. (1991). We omit the ISO spectrum between $4 \mu \mathrm{m}$ and $9 \mu \mathrm{m}$ because of a too low signal to noise ratio. An interstellar reddening of $E(B-V)=0.1$ has been applied.

\subsection{The energy balance of the system}

Several values for $T_{\text {eff }}$ of HD 161796 are quoted in the literature. Fernie (1983) quotes $6300 \mathrm{~K}$, while Hrivnak et al. (1989) use a value of $7000 \mathrm{~K}$. We use a value of 6750 , from an analysis of optical and UV photometry (van Winckel, private communication). A recent analysis of the optical absorption line spectrum however points to a somewhat higher value of $7300 \mathrm{~K}$ (van Winckel, private comm.). We estimate an uncertainty in $T_{\text {eff }}$ of about $500 \mathrm{~K}$. Using $T_{\text {eff }}=6750 \mathrm{~K}$ an extinction of $E(B-V)=0.19$ is found. This implies that $\sim 36 \%$ of the energy radiated by HD 161796 is absorbed by dust in the line of sight, assuming the standard interstellar extinction law applies (i.e. neglecting grey extinction). This extinction is caused by interstellar and/or circumstellar dust. From the infrared and millimetre observations (subtracting the stellar contribution in that range) we find that $\sim 40 \%$ of the total energy is emitted in the infrared. This suggests energy balance, which would be consistent with a spherical distribution of the dust and zero interstellar reddening. However, it is likely that a small amount of interstellar reddening exists, resulting in more IR emission from the circumstellar envelope than optical and UV absorption, thus implying a non-spherical distribution of dust. This is consistent with the observed optical and mid-infrared images that show an axisymmetric torus, typical for postAGB stars (e.g. Skinner et al. 1994; Meixner et al. 1999). Note that we cannot exclude a grey extinction component due to the presence of large grains, which would underestimate the energy absorbed in the optical and UV (see e.g. AFGL4106, Molster et al. 1999).

\section{The radiative transfer model}

As mentioned above, images of the dust surrounding HD 161796 indicate an axisymmetric geometry, and in principle a two-dimensional dust distribution is needed to fit both the images and the dust spectrum. However, we are interested in deriving quantitative mass fractions of the mineralogical components in the dust shell as well as an estimate of the mass loss history of the star. Since the ISO data show that the solid state bands are in emission and the central star shows relatively little reddening, it is reasonable to assume that the dust is optically thin. In that case, the infrared spectrum can be modeled using a spherical distribution of dust grains without losing accuracy. We note that the assumption of spherical symmetry leads to densities in the shell that are averaged over $4 \pi r^{2}$, while in reality a (significant) contrast between polar and equatorial densities may exist. We use a spherically symmetric radiative transfer model code (MODUST, Bouwman 2001) to model the complete spectrum of HD 161796. The dust is assumed to be distributed as $r^{-2}$, corresponding to a constant velocity outflow. Below we will discuss the input parameters for the model.

\subsection{The stellar parameters}

We adopt an effective stellar temperature of $6750 \mathrm{~K}$ and a distance of $1.2 \mathrm{kpc}$ (Skinner et al. 1994). The distance together with the observed flux and assumed interstellar extinction of about $E(B-V)=0.1$ (see Sect. 4) fixes the luminosity of the star to $3000 L_{\odot}$. The temperature and luminosity lead to the radius of the star, $41 R_{\odot}$. Both values are consistent with the post-AGB nature of HD 161796 but are uncertain due to the poorly determined distance to HD 161796.

\subsection{The dust components of the radiative transfer model}

We used four dust components for our radiative transfer calculations: crystalline water ice, amorphous silicates, enstatite and forsterite (see Table 1 for an overview of the optical constants used in our modeling). These components were previously identified by comparison of the ISO data to laboratory spectra of cosmic dust analogues (e.g. Molster et al. 2002a). We cannot exclude that more dust components are present (it is even likely); however, these four dust species dominate the spectrum. We assumed that all grains are spherical with a size distribution of $n(a) \propto a^{-q}$, with $q$ fixed at 3.5, which is commonly used.

The $\mathrm{H}_{2} \mathrm{O}$ ice is assumed to be in the form of a mantle surrounding an amorphous silicate core: the ice will only 
Table 1. The optical properties of the dust species used in our analysis.

\begin{tabular}{lll}
\hline \hline dust species & wavelength range $[\mu \mathrm{m}]$ & reference \\
\hline crystalline water ice & $0.1-1.3$ & Warren (1984) (compilation of amorphous ice data) \\
& $1.3-333$ & Bertie et al. (1969) (crystalline water ice at 100 K) \\
& $333-1300$ & Extrapolated \\
\hline amorphous water ice & $0.1-2.5$ & Warren (1984) \\
& $2.5-200$ & Hudgins et al. (1993) (the 10 K data) \\
& $200-1300$ & Extrapolated \\
\hline amorphous silicate & $0.1-0.4$ & Extrapolated \\
& $0.4-1300$ & Ossenkopf et al. (1992) (cool oxygen rich data) \\
\hline core-mantle grains & $0.1-0.4$ & Extrapolated \\
& $0.4-333$ & amorphous silicate and crystalline water ice data \\
& $333-1300$ & Extrapolated \\
\hline forsterite & $0.1-4.0$ & Scott \& Duley (1996) \\
& $4.0-250$ & Servoin \& Piriou (1973) \\
& $250-1300$ & Extrapolated \\
\hline enstatite & $0.1-7.5$ & Scott \& Duley (1996) \\
& $7.5-100$ & Jäger et al. (1998) $\mathrm{Mg}_{x} \mathrm{Fe}_{1-x} \mathrm{SiO}_{3}$ with $\left.x=0.96\right)$ \\
& $100-1300$ & Extrapolated \\
\hline
\end{tabular}

condense at a significant distance from the star, well outside the region where all refractory materials have been formed, using the silicates as condensation sites. If we would assume pure $\mathrm{H}_{2} \mathrm{O}$ ice grains, the low opacity of $\mathrm{H}_{2} \mathrm{O}$ ice would result in temperatures that are much lower than derived from the band strength ratios of the 43 and $60 \mu \mathrm{m}$ bands; this supports the core-mantle model. We checked the heat transfer in a $1 \mu \mathrm{m}$ core-mantle grain and found, with the thermal conductivity of crystalline water ice (Ross \& Kargel 1998), that the crystalline ice can indeed be very efficiently heated by the silicate core. However, for amorphous ice the thermal conductivity is nine orders of magnitude lower (Kouchi et al. 1992). So for amorphous water ice heating by a silicate core will not work. Crystalline water ice and amorphous water ice can therefore have very different temperatures even if both are found in the same mantles. We calculate the optical properties of a core-mantle grain with the aid of Lorentz oscillators (Bohren \& Huffman 1983). In our calculations we assumed that only a fraction of the amorphous silicates have ice mantles; we will return to this point in Sect. 4. Due to the low abundance of crystalline silicates and their low temperature, we did not include water ice mantles for this grain component, as the contribution of this water ice fraction would be negligible.

We allowed for a different upper and lower size cut-off for the amorphous and crystalline silicates. Finally, we assume co-spatial distributions for all dust species, but without thermal contact, except for the core-mantle grains.

\subsection{The optical constants of the components}

For the crystalline water ice mantle of the core-mantle grains, we used the optical constants measured by Bertie et al. (1969) extended to the UV with the compilation of measurements done by Warren (1984). The data for the amorphous water ice used to find the maximum amount possible to be hidden in the spectrum (see Sect. 4) is also taken from Warren (1984).

The optical properties of amorphous silicate are taken from the cool oxygen rich amorphous silicate data of Ossenkopf et al. (1992). The optical properties of forsterite are from Servoin \& Piriou (1973) extended to the UV with the data of Scott \& Duley (1996). The optical properties of enstatite are from Jäger et al. (1998), $\mathrm{Mg}_{x} \mathrm{Fe}_{1-x} \mathrm{SiO}_{3}$ with $x=0.96$ again extended to the UV with the data of Scott \& Duley (1996) (see also Table 1).

\section{Results and discussion of modeling}

The results of the modelling are listed in Tables 2 and 3 and the best fit is shown in Figs. 1 and 2. The best fit has been determined by eye, because several of our assumptions (spherical instead of irregularly shaped grains, only four dust species, Kurucz 1979 model) forced us to neglect some of the apparent mismatches or shifts, which cannot satisfactorily be done by a $\chi^{2}$ minimisation method. In order to estimate the errors in our modelling we changed the different parameters (even in combination) up to a point were a fit was rejected.

A fit to a spectral energy distribution from dust heated by a central star suffers from degeneracies which can only be resolved by including spatial information about the distribution of the dust (see e.g. Bouwman et al. 2000). This analysis also suffers from this difficulty, but due to the detailed spectral information the ISO data provide, we can constrain certain parameters well. Below we describe the spectral regions that were used to constrain certain model parameters.

\subsection{Limits on model parameters: Hot dust}

The temperature as a function of mass $T(M)$ of the hottest particles determines the spectral shape in the 
Table 2. Best fit model parameters. The parameters are derived using a distance of $1.2 \mathrm{kpc}$ (Skinner et al. 1994). The shell mass has been calculated with a gas to dust mass ratio of 270 and an outflow velocity of $15 \mathrm{~km} \mathrm{~s}^{-1}$ (Likkel et al. 1991). We estimate that the error on the dust mass is about 20 per cent, and on the gas mass and mass loss rate about a factor two.

\begin{tabular}{lll}
\hline \hline \multicolumn{2}{c}{ Best fit model parameters } \\
& value & at $1.2 \mathrm{kpc}$ \\
\hline Inner shell radius & $7100_{-2000}^{+600} R_{*}$ & $1400 \mathrm{AU}$ \\
Outer shell radius & $22000_{-3000}^{+1300} R_{*}$ & $4200 \mathrm{AU}$ \\
$R_{*}\left(R_{\odot}\right)$ & & 41 \\
Dust mass $\left(M_{\odot}\right)$ & & $1.7 \times 10^{-3}$ \\
Shell mass $\left(M_{\odot}\right)$ & & 0.46 \\
$\dot{M}\left(M_{\odot} / \mathrm{yr}\right)$ & $5.1 \times 10^{-4}$ \\
Interstellar $E(B-V)$ & $0.1_{-0.06}^{+0.02}$ & \\
\hline
\end{tabular}

10-20 $\mu \mathrm{m}$ wavelength range. This $T(M)$ is influenced by the inner radius and the size of the dust particles; moving the inner radius outwards and simultaneously decreasing the grain size will result in a similar spectrum. If particles become much smaller than the optical wavelength at which they absorb stellar light, their temperature and therefore their emitted spectrum becomes fairly independent of size (Bohren \& Huffman 1983). We determined an upper limit of $10^{4} R_{*}$ for the inner radius of the dust shell assuming small hot grains.

To constrain the lower size cut-off of the amorphous silicate grains we took into account the effects of grain size on the shape and strength of the $18 \mu \mathrm{m}$ amorphous silicate feature. When we lowered the lower size limit below the range indicated in Table 3 this resulted in an excess $(\sim 8 \%$ with a lower cut-off of $0.06 \mu \mathrm{m}$ ) in the region between 16 and $21 \mu \mathrm{m}$ (the amorphous silicate feature).

With this constraint on the lower size cut-off of the most abundant grains, the inner radius was found to be smaller than $7700 R_{*}$. The lower cut-off of the less abundant crystalline grains had less influence on the model spectrum and is therefore much less accurately determined.

\subsection{Limits on model parameters: Cool dust}

The outer radius should be constrained by the width of the spectral energy distribution. Increasing the outer radius will increase the width of the SED, and visa versa. However, large changes in the outer radius made relatively small changes in the width of the resulting energy distribution. Furthermore, there is a correlation between the width of the SED and the upper size cut-off of the size distribution. For an upper size cut-off of amorphous silicates larger than $6 \mu \mathrm{m}$, an excess in the 95-260 $\mu \mathrm{m}$ wavelength range was found, in addition to a widening of the SED. We could determine the outer radius of the dust shell with 30 per cent accuracy.
For crystalline silicates the upper limit to the grain size was found by looking at the width of the features, especially the forsterite complex around $33 \mu \mathrm{m}$. For an upper limit larger than $3 \mu \mathrm{m}$, the crystalline features are wider than observed. We adopted the same upper and lower grain size limits for both crystalline silicate species, i.e. 0.1 and $3.0 \mu \mathrm{m}$ respectively.

\subsection{Mass ratios}

Most accurately determined are the mass ratios of the different dust species. The strength of the different complexes, which often are dominated by one type of dust species, naturally limits the freedom in the mass ratios. We could change the water fraction of the core-mantle grains slightly, hereby changing the temperature of these grains. This changes the continuum at the longer $(>20 \mu \mathrm{m})$ wavelengths and resulted in slightly different mass fractions.

The total amount of dust could be determined within $50 \%$. The error is influenced by the relatively large error in the lower limit of the grain size. A systematic error in the total dust mass could exist if the particles are not spherical. Non-spherical particles have a different absorption coefficient per unit mass than spherical particles.

We find that about $10 \%$ of all the silicate grains are crystalline (see Table 3) a fraction which is not uncommon for oxygen-rich circumstellar envelopes (Molster 2000). The derived mass-averaged temperature for crystalline water ice is found to be $\sim 70 \mathrm{~K}$ and amorphous silicates are about $110 \mathrm{~K}$. Crystalline pyroxene grains were found to be about $15 \mathrm{~K}$ warmer than forsterite grains, which is in agreement with the results found by Molster (2000).

\subsection{Comparison of model parameters with imaging}

The inner and outer shell radius of our best fit model agree well with the observations. We adopt a distance of $1.2 \mathrm{kpc}$, resulting in an outer radius of the dust shell of 3.5 arcsec. This is consistent with the upper limit of 6.5 arcsec derived from CO observations by Bujarrabal et al. (1992) and equal to the size of $4.34^{\prime \prime} \times 2.46^{\prime \prime}$ derived from the optical observations of the scattered light by Ueta et al. (2000). We note that the outer radius is mostly determined by the position of the cold dust. In a non-spherical (torus) distribution of the dust, the shielding in the equatorial plane can become very efficient and thus the dust colder than we have derived from our 1-D analysis. This might bring the outer radius closer to the star. Because this requires a $2-\mathrm{D}$ modeling we did not investigate this further.

The inner radius of the best fit model is 1 arcsec, which is about twice the value of 0.4 arcsec estimated by Skinner et al. (1994) based on $N$ band images. Two effects will likely explain this apparent discrepancy. First, we assumed spherical symmetry, while as discussed before, the real shape is more likely to be a torus. A smaller angular size of the inner radius of the dust shell may partially be a 
Table 3. Mass fractions, size range and temperature ranges of the best fit model. The core sizes of the core-mantle grains are equal to the sizes of the amorphous silicates without ice. The temperature ranges reflect the extremes at the inner and outer boundaries of the dust shell. The uncertainty in the derived temperatures is about 10 per cent.

\begin{tabular}{lllll}
\hline \hline Dust type & $\begin{array}{l}\text { dust mass } \\
{[\%]}\end{array}$ & $\begin{array}{l}\text { min. particle } \\
\text { size }[\mu \mathrm{m}]\end{array}$ & $\begin{array}{l}\text { max. particle } \\
\text { size }[\mu \mathrm{m}]\end{array}$ & $\begin{array}{l}\text { Temperature } \\
\text { range }[\mathrm{K}]\end{array}$ \\
\hline Am. silicates & 58 & 0.18 & 6 & $137-47$ \\
Core-mantle grains & 32 & 0.5 & 17 & $75-37$ \\
$\begin{array}{c}\text { Am. silicates } \\
\text { Cryst. } \text { water } \text { ice }\end{array}$ & 5 & - & - & - \\
Enstatite & 27 & - & - & - \\
Forsterite & 6 & 0.1 & 3 & $76-45$ \\
\hline
\end{tabular}

projection effect. Furthermore, we assumed spherical particles in our calculations. Non-spherical particles have a relatively larger surface area. Therefore, real particles are likely to be a little colder (at the same distance) than the ones in our calculations and are therefore expected to be closer to the central star.

The $E(B-V)$ of the full radiative transfer model is only 0.06 , which is less than the value of 0.19 determined from the stellar SED, implying an interstellar $E(B-V)$ of order 0.1 (see Fig. 2).

\subsection{Discussion}

We included both "bare" amorphous silicate grains and core-mantle grains consisting of an amorphous silicate core and a crystalline $\mathrm{H}_{2} \mathrm{O}$ ice mantle. If all silicates would be equally covered with an $\mathrm{H}_{2} \mathrm{O}$ ice mantle, the strength of the 43 and $60 \mu \mathrm{m}$ bands can only be fitted by using relatively thin mantles. Thin mantles on top of large cores result in too high temperatures of the core-mantle grains. We resolved this discrepancy by assuming that only a fraction of the silicates have a relatively thick mantle. While in a spherical shell the co-existence of bare and core-mantle grains may be hard to explain, the torus geometry of the dust shell surrounding HD 161796 provides a natural spatial separation of these two components, with the coremantle grains concentrated more in the equatorial regions of the torus.

Kouchi et al. (1992) measured the speed of amorphization of crystalline water ice under UV radiation. The UV radiation field around HD 161796 would under normal conditions transform the crystalline water ice into amorphous water ice within a day. So, we must conclude that the crystalline water-ice is efficiently shielded from the harsh UV radiation field, for example by the density enhancement in the torus. This again leads to the conclusion that most if not all of the crystalline water ice is located in the torus.

Our fit shows a small offset for the $43 \mu \mathrm{m}$ and a larger offset for the $60 \mu \mathrm{m}$ water ice features. Part of these errors are due to the fact that the measurements of the optical constants from Bertie et al. (1969) were done at $100 \mathrm{~K}$ and at a poor spectral resolution. Higher spectral resolution measurements at the right temperature of $\sim 70 \mathrm{~K}$ would give a better fit as the features tend to move to shorter wavelength when measured at lower temperatures. The large discrepancy at $60 \mu \mathrm{m}$ is not solved using the $70 \mathrm{~K}$ data from Smith et al. This indicates that additional solid state components may contribute in this wavelength range, such as diopside $\left(\mathrm{CaMgSiO}_{3}\right.$, Koike et al. 2000) or dolomite $\left(\mathrm{CaMg}\left(\mathrm{CO}_{3}\right)_{2}\right.$, Kemper et al. 2002b).

Our best fit model shows a weak absorption at $3.1 \mu \mathrm{m}$ due to $\mathrm{H}_{2} \mathrm{O}$ ice, which is not observed (see Fig. 3). This indicates that we have slightly overestimated the amount of $\mathrm{H}_{2} \mathrm{O}$ ice in the line of sight towards the star. This may have several reasons: (1) blends of the 43 and $60 \mu \mathrm{m}$ ice bands with other species (see above), or (2) deviations from spherical symmetry.

We also investigated the possibility that amorphous $\mathrm{H}_{2} \mathrm{O}$ ice is present. Amorphous $\mathrm{H}_{2} \mathrm{O}$ ice has a broad resonance near $45 \mu \mathrm{m}$ but lacks the $60 \mu \mathrm{m}$ band. We find that about $10 \%$ of the dust mass could be pure amorphous water ice (or core-mantle) at the expense of slightly decreasing the mass of the crystalline $\mathrm{H}_{2} \mathrm{O}$ ice (see Fig. 4). We note that the amorphous $\mathrm{H}_{2} \mathrm{O}$ ice is very cold due to its poor conductivity and mainly contributes in the 60$100 \mu \mathrm{m}$ wavelength range.

With the amount of water ice found we can derive a estimate of the lower limit for the gas to dust mass ratio. We find that about $27 \%$ of all dust mass in the shell consists of water ice (see Table 3). An estimate of the water fraction per mass, can be derived from the water vapour fraction by number relative to $\mathrm{H}_{2}$. González-Alfonso \& Cernicharo (1999) found that the water fraction by number in oxygen rich circumstellar environments is about $1-2 \times 10^{-4}$. We will use $1.5 \times 10^{-4}$ and an average mass for the atoms in outflow of an AGB star (mainly $\mathrm{H}$ and He) to be $1.4 \mathrm{~m}_{\mathrm{p}}$, with $m_{\mathrm{p}}$ the proton mass. This makes the mass fraction of water in an outflow $\left(1.5 \times 10^{-4} \times 18 /(2 \times 1.4)\right) \sim 1 \times 10^{-3}$ and gives for HD 161796 a gas to dust mass ratio after the water ice condensation of $\sim 270$. Since we find a water ice mass of $27 \%$ this makes the gas to dust ratio before the ice condensation $\sim 370$. This number is a lower limit since we assumed in deriving this number that all water vapour present in the outflow has become ice, which does not have to be the case.

Based on the inner and outer radius of the dust shell (See Table 2), the distance of $1.2 \mathrm{kpc}$ (Skinner et al. 1994) 


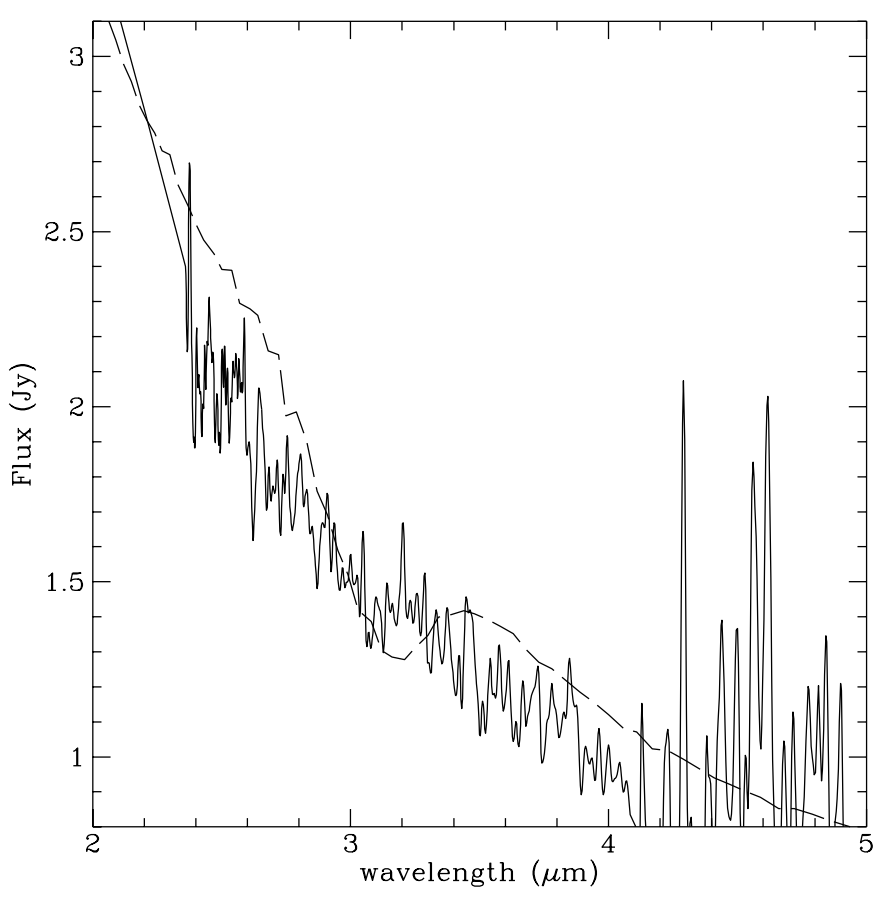

Fig. 3. A detail of the best fit of the full radiative transfer model (dashed line) compared to the spectrum of HD 161796 (solid line). A $3.1 \mu \mathrm{m}$ absorption feature of water ice is visible in the model. The small apparent absorption in the measured spectrum near $3 \mu \mathrm{m}$ is caused by an anomaly in the measured spectrum.

and the outflow velocity of $15 \mathrm{~km} \mathrm{~s}^{-1}$ (Likkel et al. 1991) we find that the mass loss episode stopped about 430 years ago after it had lasted for about 900 years. In this period a total dust mass of about $1.7 \pm 0.4 \times 10^{-3} M_{\odot}$ has been lost. This results in a mean dust mass loss rate of $1.9 \times$ $10^{-6} M_{\odot} \mathrm{yr}^{-1}$. With the assumed gas to dust ratio of 270 after the water condensation, we find a total shell mass of $(0.46 \pm 0.1) \quad M_{\odot}$ and a total mass loss rate of $5.1 \times 10^{-4} M_{\odot} \mathrm{yr}^{-1}$. The uncertainty in the mass loss rate is about a factor 2 .

\section{The formation of ice mantles}

The most prominent spectral bands in the ISO spectrum of HD 161796 are the crystalline $\mathrm{H}_{2} \mathrm{O}$ ice features. The presence of ice mantles on the silicate grains poses restrictions on possible models. Water molecules can only condense onto the grains in significant amounts if the grains are cold and the gas densities are high enough for water ice to be stable. In this section we will quantify this constraint.

We assume that the ice condenses on pre-existing silicate grains. Therefore, the process of nucleation (seed formation) is not relevant. In a gas-grain mixture, gas molecules collide with grains because of thermal motions and because of drift motions between gas and dust. For the estimates in this section we assume that the relative speed of gas and dust particles is given by a collision speed $v_{\text {coll }}$. We also assume that the grain is cold, so that the

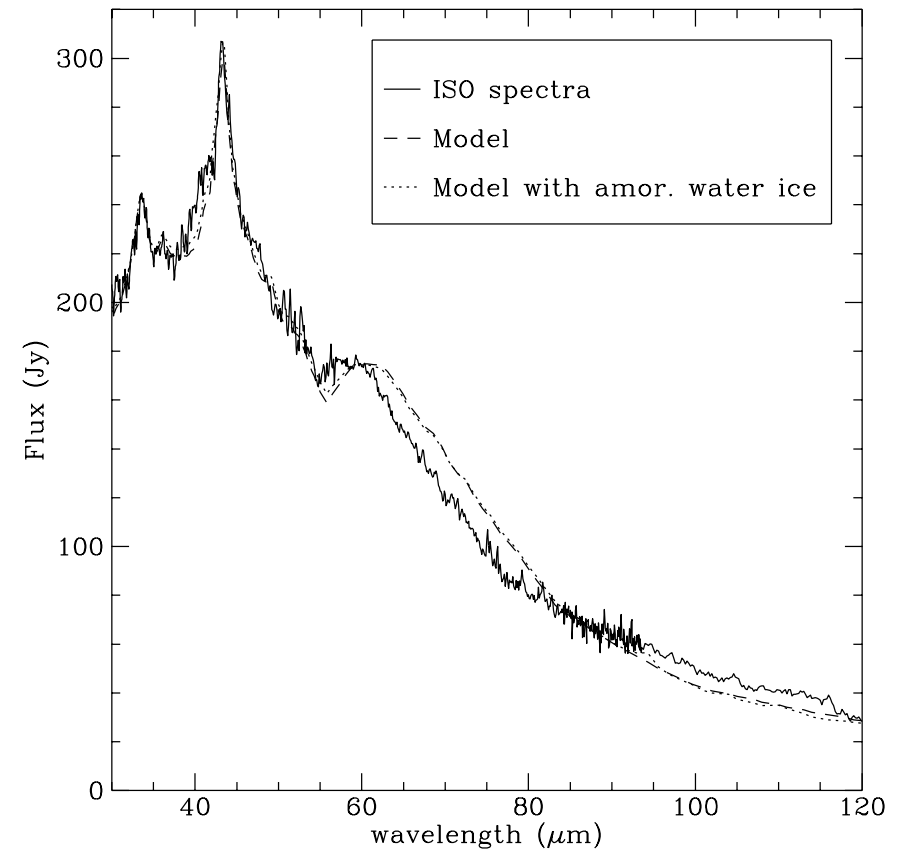

Fig. 4. The full radiative transfer model with $11 \%$ of amorphous water ice (dotted line) compared with the preferred model (dashed line) and the observed spectrum (solid line). The grain size of the pure amorphous water ice grains is the same as the core-mantle grains. This way we model that some grains have an amorphous water ice mantle which is thermally insulated from the grain, but has an equal size as the crystalline grains. The amorphous ice data used is the $10 \mathrm{~K}$ data from Hudgins et al. (1993).

evaporation of ice molecules from the grain can be neglected. The rate of change of the particle radius, $\mathrm{d} a / \mathrm{d} t$ is then given by

$\frac{\mathrm{d} a}{\mathrm{~d} t}=\frac{V_{\mathrm{H}_{2} \mathrm{O}}}{4 \pi a^{2}} \nu_{\text {coll }}$

where $V_{\mathrm{H}_{2} \mathrm{O}}=18 m_{\mathrm{H}} / \rho_{\text {ice }}$ is the volume occupied by an $\mathrm{H}_{2} \mathrm{O}$ molecule in ice. $m_{\mathrm{H}}$ is the mass of an hydrogen atom and $\nu_{\text {coll }}$ is the collision frequency between gas and dust given by

$\nu_{\text {coll }}=\pi a^{2} n_{\mathrm{H}_{2} \mathrm{O}} v_{\text {coll }}$

where $n_{\mathrm{H}_{2} \mathrm{O}}$ is the number density of water molecules in the gas phase. After inserting Eq. (2) into Eq. (1) we can immediately integrate and find the change of the radius of a dust grain $\Delta a$ due to ice condensation in a given time interval $\Delta t$ :

$\Delta a=\frac{1}{4} V_{\mathrm{H}_{2} \mathrm{O}} n_{\mathrm{H}_{2} \mathrm{O}} v_{\mathrm{coll}} \Delta t$.

Note that we have assumed that both $v_{\text {coll }}$ and $n_{\mathrm{H}_{2} \mathrm{O}}$ are independent of time, in particular we have neglected the depletion of water vapor. We can now estimate which density of water vapor molecules is needed to achieve ice mantles of a given thickness in a given time:

$n_{\mathrm{H}_{2} \mathrm{O}}=\frac{392}{\mathrm{~cm}^{3}} \frac{\Delta a}{1 \mu \mathrm{m}} \frac{1000 \mathrm{yr}}{\Delta t} \frac{\rho_{\text {ice }}}{0.93 \mathrm{~g} / \mathrm{cm}^{3}} \frac{10 \mathrm{~km} \mathrm{~s}^{-1}}{v_{\text {coll }}}$. 
The $10 \mathrm{~km} \mathrm{~s}^{-1}$ for the collision velocity is higher than the thermal speed at the temperatures where water ice is stable $(<140 \mathrm{~K})$. However, the higher value accounts for a possible drift between dust and gas, induced by radiation pressure from the star (Dominik et al. 1989). Using a water vapor abundance of $10^{-4}$ (González-Alfonso \& Cernicharo 1999) we find that the required hydrogen density in the region where the ice mantles formed is $\approx 3.9 \times 10^{6} \mathrm{~cm}^{-3}$. Using the current location of the core-mantle grains of about $3000 \mathrm{AU}$ (1200 AU) and an expansion velocity of $10 \mathrm{kms}^{-1}$ we find that the required mass loss rate in a spherical outflow is $\dot{M} \simeq 6 \times 10^{-3} M_{\odot} /$ yr, which is much too high. However, the ice mantles were probably grown in a much earlier phase when the star was still on the AGB. For a star with a high mass loss rate, the location of the $140 \mathrm{~K}$ dust temperature in the shell is approximately at $300 \mathrm{AU}$ (Kemper, private comm.) If this was indeed the case, the mass loss rate required to form the ice mantles in 1000 yrs is

$\dot{M} \simeq 6 \times 10^{-5} \frac{M_{\odot}}{\mathrm{yr}}$.

The mass loss rate derived in Eq. (5) is a lower limit to the required mass loss rate to form $\mathrm{H}_{2} \mathrm{O}$ ice, since we have ignored the depletion of $\mathrm{H}_{2} \mathrm{O}$ from the gas phase as the mantles grow, and we have ignored the density gradient in the shell in the $\mathrm{H}_{2} \mathrm{O}$ ice growth region. The high mass loss rate required to form $\mathrm{H}_{2} \mathrm{O}$ ice is consistent with the observed very high mass loss rate.

\section{Conclusions}

The high quality ISO spectrum, with its wide wavelength coverage, allows a detailed analysis of the dust composition of this high latitude post-AGB star. We find a modest amount (10 per cent) of crystalline silicates, which is typical for similar stars (e.g. Molster et al. 1999; Kemper et al. 2002a). These crystalline silicates are Fe-poor, in contrast to the amorphous silicates.

The ISO spectrum of HD 161796 is unique in that it shows very strong crystalline $\mathrm{H}_{2} \mathrm{O}$ bands. This must imply that the conditions to form $\mathrm{H}_{2} \mathrm{O}$ ice in the envelope were very favourable, compared to other oxygen-rich AGB stars. We showed that core-mantle grains, consisting of a silicate core and a water ice mantle, must exist, since this is the only way to get the water ice at the right temperature without assuming a strange geometry of the dust shell.

The critical parameter which determines the formation of $\mathrm{H}_{2} \mathrm{O}$ ice is the density of water vapour at the condensation point in the outflow. It is possible that the $\mathrm{H}_{2} \mathrm{O}$ condensation radius in $\mathrm{HD} 161796$ was smaller than in other AGB stars, leading to efficient $\mathrm{H}_{2} \mathrm{O}$ ice deposition. In view of the high mass loss rate of HD 161796, and considering the modest luminosity of HD 161796 compared to typical $\mathrm{OH} / \mathrm{IR}$ stars, the density in the outflow may have been substantially higher and the ice condensation radius accordingly smaller at high densities. From the preference of water ice to form in a high density regime we expect that most water ice is present in the torus. Since crystalline water ice becomes amorphous when exposed to UV radiation, the torus is also the likely place where the crystalline water ice can survive.

We find that the mass loss episode of HD 161796 lasted for about 900 years and stopped about 430 years ago. In this period a total dust mass of about $1.7 \times 10^{-3} M_{\odot}$ has been lost. This results in a mean dust mass loss rate of $1.9 \times 10^{-6} M_{\odot} \mathrm{yr}^{-1}$. We were able to derive a lower limit to the gas to dust ratio of 270 from the amount of water ice. A total shell mass of $0.46 M_{\odot}$ and a total mass loss rate of $5.1 \times 10^{-4} M_{\odot} \mathrm{yr}^{-1}$ result. These numbers are similar to those found by e.g. Skinner et al. (1994).

In order to fit the spectrum we had to assume a particle size distribution which lacks small grains: the grains around HD 161796 are (on average) much larger than in the ISM. A similar result was obtained by Molster et al. (1999) for AFGL4106.

Acknowledgements. We would like to thank R. Sylvester for the use of his reduced LWS spectrum of HD 161796 and H. van Winckel for his measurement of the reddening. FJM acknowledges support from NWO under grant 781-71-052 and under the talent fellowship program. LBFMW, AdK and CD acknowledge financial support from NWO "Pionier" grant number 616-078-333. This work was partly supported by NWO Spinoza grant 08-0 to E. P. J. van den Heuvel.

\section{References}

Barlow, M. J. 1998, Ap\&SS, 255, 315

Bertie, J. E., Labbé, H. J., \& Whalley, E. 1969, J. Chem. Phys., 50,4501

Bohren, C. F., \& Huffman, D. R. 1983, Absorption and scattering of light by small particles (New York: Wiley)

Bouwman, J. 2001, Ph.D. Thesis, University of Amsterdam

Bouwman, J., de Koter, A., van den Ancker, M. E., \& Waters, L. B. F. M. 2000, ASP Conf. Ser. 196, Thermal Emission Spectroscopy and Analysis of Dust, Disks, and Regoliths, 63

Bujarrabal, V., Alcolea, J., \& Planesas, P. 1992, A\&A, 257, 701

Clegg, P. E., Ade, P. A. R., Armand, C., et al. 1996, A\&A, 315, L38

de Graauw, T., Haser, L. N., Beintema, D. A., et al. 1996 A\&A, 315, L49

Dominik, C., Gail, H.-P., \& Sedlmayr, E. 1989, A\&A, 223, 227

Fernie, J. D. 1983, ApJ, 265, 999

Fernie, J. D., \& Garrison, R. F. 1984, ApJ, 285, 698

González-Alfonso, E., \& Cernicharo, J. 1999, ApJ, 525, 845

Hrivnak, B. J., Kwok, S., \& Volk, K. M. 1989, ApJ, 346, 265

Hudgins, D. M., Sandford, S. A., Allamandola, L. J., \& Tielens, A. G. G. M. 1993, ApJS, 86, 713

Jäger, C., Molster, F. J., Dorschner, J., et al. 1998, A\&A, 339, 904

Justtanont, K., Barlow, M. J., Skinner, C. J., \& Tielens, A. G. G. M. 1992, ApJ, 392, L75

Kemper, F., de Koter, A., Waters, L. B. F. M., Bouwman, J., \& Tielens, A. G. G. M. 2002a, A\&A, 384, 585 
Kemper, F., Jäger, C., Waters, L. B. F. M., et al. 2002b, Nature, 415, 295

Molster, F. J., Waters, L. B. F. M., Tielens, A. G. G. M., \& Barlow, M. J. 2002b, A\&A, 382, 184

Kessler, M. F., Steinz, J. A., Anderegg, M. E., et al. 1996, A\&A, 315, L27

Molster, F. J., Waters, L. B. F. M., Trams, N. R., et al. 1999, A\&A, 350, 163

Koike, C., Tsuchiyama, A., Shibai, H., et al. 2000, A\&A, 363, 1115

Ossenkopf, V., Henning, T., \& Mathis, J. S. 1992, A\&A, 261, 567

Kouchi, A., Greenberg, J. M., Yamamoto, T., \& Mukai, T. 1992, ApJ, 388, L73

Kurucz, R. L. 1979, ApJS, 40, 1

Likkel, L. 1989, ApJ, 344, 350

Parthasarathy, M., \& Pottasch, S. R. 1986, A\&A, 154, L16

Ross, R. G., \& Kargel, J. S. 1998, in ASSL Vol. 227, Solar System Ices, 33

Scott, A., \& Duley, W. W. 1996, ApJS, 105, 401

Likkel, L., Forveille, T., Omont, A., \& Morris, M. 1991, A\&A, 246, 153

Meixner, M., Ueta, T., Dayal, A., et al. 1999, ApJS, 122, 221

Molster, F. J. 2000, Ph.D. Thesis, University of Amsterdam, The Netherlands

Molster, F. J., Waters, L. B. F. M., \& Tielens, A. G. G. M. 2002a, A\&A, 382, 222

Servoin, J. L., \& Piriou, B. 1973, Phys. Stat. Sol., 55, 677

Skinner, C. J., Meixner, M. M., Hawkins, G. W., et al. 1994, ApJ, 423, L135

Ueta, T., Meixner, M., \& Bobrowsky, M. 2000, ApJ, 528, 861 Walmsley, C. M., Chini, R., Kreysa, E., et al. 1991, A\&A, 248, 555

Warren, S. G. 1984, Appl. Opt., 23, 1206 